\title{
Métodos y Técnicas de investigación Cualitativa y Cuantitativa en Geografía
}

Fredis Mateo Aguilar Herrera Docente de la Universidad Pedagógica Nacional Francisco Morazán

Fredisaguilar@yahoo.com

\section{Resumen}

La temática abordada en relación a los métodos y técnicas de investigación cualitativa y cuantitativa en geografía tiene brinda a los lectores un esbozo general a nivel descriptivo de estos paradigmas metodológicos aplicados en el campo de las ciencias naturales y sociales, asimismo establecer una caracterización teórica y de manera ejemplificada dichas metodologías y técnicas con el objetivo que los investigadores en temas geográficos comprendan y determinen a su vez una de ellas.

\section{Palabras Clave:}

Métodos y técnicas cualitativas, métodos y técnicas cuantitativas, investigación, cambios paradigmáticos, fenomenología en la geografía, definición del problema, técnicas de autoafirmación, recogida de datos y análisis de datos.

\section{Introducción}

El presente artículo tiene como propósito llenar el vacío bibliográfico en temática de investigación geográfica en el país, además orientar a todos los profesionales de la geografía y de ciencias afines a las ciencias geoespaciales interesados en el estudio de estas áreas temáticas de metodologías de investigación desde la ciencia geográfica. Por otro lado es importante que los que se atreven a 
hacer investigación conozcan las bases epistemológicas de la misma y posteriormente ser aplicada. Lo que implica que antes de la selección de un tema de investigación, lo primero que hay que hacer es identificar los efectos de una determinada realidad observada para posteriormente identificar las posibles causas, para convertirlo en el problema de investigación, por medio de interrogantes planteadas, como de técnicas, tipo y diseño de investigación acorde a la metodología previamente seleccionada. Otras de las finalidades de adentrarnos en esta temática es porque en la Universidad Pedagógica Nacional Francisco Morazán se han identificados los espacios pedagógicos tanto en el pregrado como el postgrado en el cual es posible realizar la investigación temas de orden geográfico.

\section{Métodos Técnicas Cualitativas}

La investigación cualitativa comenzó a utilizarse por los años sesenta, principalmente en Estados Unidos e Inglaterra, fue a partir de los años ochenta cuando tomó auge para la investigación de problemas en el campo educacional. Muñoz (2011:6)

El interés de la investigación con datos de tipo cualitativo se profundiza desde la década de los setenta, por considerar que no es posible captar la riqueza y profundidad de los estudios geográficos, utilizando técnicas y métodos de corte neopositivista. Sin embargo desde la antigüedad y a lo largo de la evolución de la ciencia geográfica, han existido estudios de carácter cualitativo basado en la observación y trabajos de campo de viajeros desde Herodoto, que recoge elementos territoriales básicos que tienen que ver con las diferencias étnicas, con las particularidades sociales, con las singularidades y regularidades del espacio, estudia al mismo como estrategia de poder, Estrabón, su discurso aparece como interpretación renovada de la Geografía, por lo que trata de una reflexión sobre la naturaleza y el significado de la representación geográfica, Marco Polo, considerado uno de los grandes explorados europeos que viajaron al oriente asiático y que a su vez narró sus experiencias expedicionarias, Alejandro Von Humbotlh, quien 
describió con detalle formas del relieve y condiciones climáticas, indicando con precisión y acierto las relaciones entre estos dos factores físicos y estudia la distribución de plantas y animales en un determinado espacio geográfico, Karl Ritter, establece la moderna Geografía Física científica, introduce el estudio de las relaciones del medio físico y hombre, Federico Ratzel, estudia por medio de los mapas, la manera como los hombres se hallan distribuidos y agrupados en la tierra y a la vez explica el por qué de esta distribución y reparto de acuerdo con los movimientos de los pueblos que se producen a lo largo de la historia, asimismo estudia los efectos que el medio físico pueden producir en los individuos y sociedades, Vidal de la Blache, interesado por estudios de región.

La geografía como disciplina científica y académica ha experimentado notables cambios teóricos, metodológicos, paradigmáticos y enfoques investigativos a lo largo del devenir histórico. Por tanto hoy en día la geografía ha dejado de ser una ciencia meramente cuantitativa y ha pasado a ser una ciencia reflexiva e interpretativa, mediante la aplicación de métodos y técnicas cualitativas sobre todo en los que corresponde al estudio de los fenómenos humanos en relación a los fenómenos físicos.

En la introducción en geografía de métodos y técnicas cualitativas ha jugado un papel un papel decisivo la fenomenología. Reciente Milton Santos (1995), la considera como un instrumento fundamental dentro de la nueva disciplina, ya que permite " a través de las cosas, de los objetos, es decir, de la configuración geográfica... pasar de lo universal a lo particular, sin caer en el riesgo de una interpretación empiricista, yendo más allá de la cosa, del objeto, de la materialidad del espacio" y, en suma recuperar la dicotomía entre objetividad y subjetividad. García (1998:16)

El estudio de la fenomenología en la geografía implica la comprensión explicativa de la relación o interacción de los fenómenos humanos y físicos desde la perspectiva que tiene cada actor acorde a sus creencias, conocimientos, experiencias y valores. 
Es así que la percepción que tiene cada sociedad o comunidad sobre entorno o de un determinado espacio varía.

Es así que empleando una investigación geográfica de naturaleza cualitativa debe preguntarse por ejemplo ¿ por qué un determinado asentamiento humano se instaló en una terraza aluvial o cualquier zona de riesgo natural y por qué no en otro lugar?. ¿ Por qué un sector de mismo lugar de Honduras hay mayor desnutrición y por qué en otro sector no lo hay?. Por lo que para responder a dichas preguntas se requiere necesariamente de observaciones de campo del área de estudio, como también la aplicación de encuestas de tipo cualitativo y otras técnicas documentales y literarias que proporcionen información para la explicación de dicho fenómeno geográfico.

Además es necesario recurrir a la geografía de la percepción, puesto que un mismo fenómeno físico o humano tiene distintas apreciaciones. La metodología cualitativa no comienza con un conjunto de hipótesis que hay que verificar, sino el reconocimiento de la del área de estudio, mediante el planteamiento de una serie de problemas y los objetivos de la investigación.

La importancia de la aplicación de metodología cualitativa en geografía es que produce datos descriptivos al utilizar las propias palabras habladas o escritas de las personas y de sus comportamientos directamente observados. La metodología cualitativa, es humanista, inductiva, empática, flexible e interactiva entre el investigador y las personas objeto de estudio.

Como señala Ruiz e Ispizua (1989) la investigación que utiliza métodos y técnicas cualitativas supone un proceso análogo al empleado en otros tipos de investigación y que en síntesis pasa por cinco fases: definir el problema, diseño de la investigación, recoger los datos, analizarlos y finalmente validar la investigación y exponer sus resultados. García Ballesteros y otros (1998:20). 
La definición del problema se orienta a la búsqueda de motivaciones y significados, entrando en contacto con el mismote forma abierta, para posteriormente concretar la situación objeto de estudio con sus parámetros espaciales, temporales y sociales. Como señala Ruiz e Ispizua (1989) definir el problema objeto de estudio no es delimitar, rodear, circunscribir con precisión, sino situarse, orientarse, sumergirse, acercarse, contactar con el núcleo del foco el centro del problema.

El diseño de investigación cualitativa, consiste en la selección de pocas personas a estudiar (puede ser expertos, personas o grupos previamente seleccionados), pero con la intención de obtener datos reales, por medio de la interrelación socio-espacial que permitan un estudio a profundidad y la comprensión de motivaciones, valores y significados, no importando el tamaño muestral como en las investigaciones cuantitativas, cuyos resultados son generalizados.

Una vez diseñada la investigación, se recurre a la recogida de datos. Las técnicas utilizadas según Clemente y Gil (1985), se clasifican en tres grandes grupos: técnicas de autoafirmación, técnicas de observación y técnicas documentales. García Ballesteros y otros (1998:21).

Las técnicas de autoafirmación obtienen los datos a partir de informes orales o escritos de los propios sujetos investigados. En geografía se utiliza la entrevista en profundidad, en las que pueden haber o no un guión de los temas a tratar, pero que en todos los casos los sujetos tienen libertad de responder su modo, minimizando la instrucción del investigador, además se aplica la técnica Delphí, que consiste en un análisis del estado opinión de carácter prospectivo, relacionado con temas que tenga que ver con la planificación territorial, previsiones de flujos comerciales y transporte público, estudios medioambientales urbanísticos, vivienda e infraestructura vial, entre otros, que pretendan cambios novedosos y la mejor toma de decisiones en un determinado territorio. 
También se emplea la técnica de grupos de discusión, como una técnica no directiva en dónde se lanza a discusión un determinado tema de índole socio-espacial, en donde se analiza la realidad actual y se modela la realidad futura de un determinado espacio, ejemplo: analizar la situación problemática de causa y efectos de contaminación ambiental en una determinada corriente hidrográfica y proponer las posibles soluciones.

A este grupo de técnicas de autoafirmación tenemos las historias de vida y autobiografías asistidas, mediante las cuales las personas narran la situación problemática de un determinado fenómeno humano en correlación de un fenómeno físico. En la primera de ellas el entrevistador interfiere mínimamente, mientras que en la segunda el investigador colabora en la reconstrucción de los significados de la historia de vida, ejemplo: ¿por qué emigró del campo a la ciudad?. Asimismo se puede hacer uso de las escalas de autoevaluación para valorar el significado de la emigración hacia los Estados Unidos de América en una escala delimitada así: ventaja _ desventaja ; difícilfácil; motivado-obligado; contento- arrepentido; empeoró progresó.

Las técnicas de observación, más aplicadas en geografía son la tipo participante, en la que el investigador obtiene los datos al inmiscuirse en el grupo estudiado, pudiendo de esta manera registrar las relaciones socio-espaciales. Mientras que las técnicas documentales, comprende producciones literarias, archivos públicos y prensa escrita.

Posteriormente pasamos al análisis de lo datos, mediante la exploración, clasificación, codificación y categorización de los mismos, para poder brindar una mejor explicación del problema estudiado. Una vez realizado el análisis de datos se procede a la etapa final de la estructuración del informe final y por ende la socialización y validación de los resultados.

Para Muñoz Campos (2000:15), la investigación cualitativa tiene 
como características : es subjetiva (los datos son filtrados por criterio del investigador), las reglas de procedimiento no son precisas, ya que no específica previamente el método de recogida de datos. Se basa más en la intuición, es holística, ya que abarca el fenómeno que estudia en su conjunto. No estudia las variables por separado, es recursiva, ya que el método de investigación es emergente, pues se va elaborando a medida avanza la investigación, el análisis estadístico que emplea no es riguroso, ya que a lo sumo solo llega a hacer recuentos de frecuencia y categorizaciones o clasificaciones, no se puede replicar, casos como el un terremoto, consecuencias de una guerra, derribamiento de torres gemelas, hundimiento de un buque petrolero en el océano y las conclusiones no son genenarizables.

\section{Métodos y Técnicas Cuantitativas}

Según Chávez y Pérez (2005), citado por Martínez y Bernaza, investigación cuantitativa (empírico- analítica) se sustenta en el positivismo, neopositivismo y el pragmatismo. Esta orientación de la investigación científica resulta ser la más utilizada en las ciencias sociales.

La misión de la investigación científico- cuantitativa es explicar los fenómenos, por interesarse en las causas que originan éstos (principio de verificación) y se apoya en las técnicas estadísticas para el procesamiento de la información, la que se obtiene mediante los métodos empíricos: la observación, la encuesta, la entrevista y el experimento; y de esa manera llegar a las conclusiones que son altamente generalizables.

Entre tanto, Ortez (2000, 60), expresa que la investigación cuantitativa le interesa las mediciones del fenómeno o proceso, ejemplo: con qué frecuencia ocurre, cual es el porcentaje, cuántos lo dicen. Sus mayores exponentes son la investigación descriptiva, analítica, empírica y la experimental. Dentro de los métodos y técnicas cuantitativas, uno de los aspectos más sobresalientes es la amplia 
incorporación a la investigación de las mediciones y experimentaciones en el campo y en el laboratorio.

Según Anhert (1980) debe hablarse de mediciones observacionales y de mediciones experimentales. El objeto de las primeras es obtener valores de los parámetros naturales sin interferir con el medio, pues se pretende cuantificar la tasa del proceso observado, como por ejemplo la observación y medición de movimientos de masa de tierra. Sanjaume (199: 107)

Las mediciones experimentales en cambio intentan soslayar el "ruido" u obtener resultados sobre un número muy limitado pero preciso de sus elementos y de sus relaciones; sus resultados se requiere que sean útiles para la identificación o la formulación de reglas o leyes generales. Por tanto no se debe confundir una medición por precisa que sea con un experimento, aunque la evaluación de este nos sea absolutamente precisa. Así que no todo trabajo llevado a cabo en un laboratorio es experimental, como puede ser el caso de la medición granulométrica de sedimentos, del establecimiento de composición química, etc. En cambio si constituyen experimento geomorfológicos tanto de campo como de laboratorio el estudio de simulación de lluvia, de distintos procesos de erosión ( impacto de las gotas de lluvia, escorrentía superficial, etc.) o el estudio del comportamiento fluvial mediante la utilización de lechos artificiales.

La geografía cuantitativa, está orientada a la construcción de teorías, leyes y prueba de hipótesis con base a análisis estadísticos y cartográficos del espacio, concebido éste como concepto abstracto cuantificable.

Según Muñoz Campos (2000:15), la investigación cuantitativa tiene como principales características: aplicación método hipotéticodeductivo, la representación de muestras representativas, medición objetiva de variables, utilización de técnicas de recogida de datos de datos cuantitativos con instrumentos como son los cuestionarios, 
las escalas, los test, aplicación estadística en el análisis de datos y procura la prueba de hipótesis y teorías.

\section{Conclusiones}

* El investigador, al momento de seleccionar de la metodología de investigación, debe de saber si el propósito de la investigación es pura o aplicada, debe saber el lugar dónde se realizará, puede ser bibliográfica, de campo o de laboratorio, debe de saber los niveles de profundidad en relación si es explotaría, descriptiva, explicativas, etnográfica, etc., y debe saber si el tratamiento de las variables son experimentales, no experimentales o cuasi experimentales.

* Por lo tanto la metodología de investigación se determina, tomando en consideración la realidad a estudiar, la forma como se hacer, tipo de información existente y la que se desea generar y a los resultados a los que se quiere llegar.

* Esta pluralidad de la investigación científica, ha provocado a lo largo tiempo una discusión epistemológica sobre la selección de la metodología cualitativa y cuantitativa, en el sentido de verlas como opuestas o por separadas, sin embargo en la actualidad se ha logrado verla como complementarias en el adecuado tratamiento de datos de manera mixta.

* Las investigaciones con metodología cualitativa y cuantitativa, son factibles a lo interno de la Universidad Pedagógica Nacional Francisco Morazán, ya que en el pregrado en la Carrera del Profesorado de Ciencias Sociales en el grado de Licenciatura, en su plan de estudios, existen los espacios formativos de: Fundamentos de Geografía, Geografía Física, Geografía Humana, Geografía de Honduras, Geografía Mundial, Metodología de Investigación Cuantitativa, Taller de Estadística Descriptiva Aplicada a la Investigación, Metodología y en el Postgrado se cuenta con la Maestría en la Enseñanza de la Geografía con 
orientación en Ordenamiento Territorial, en Población Ambiente y Desarrollo y su plan de estudios, se tiene un tola de 13 módulos de la especialidad y un módulo de Taller de tesis.

* Estos distintitos espacios formativos existentes en la UPNFM, muestran el escenario multidimensional de diferentes temáticas en el que pueden desarrollar múltiples y diversas investigaciones en correspondencia de las Líneas de Investigación y Postgrado, Líneas de Investigación de la Maestría en la Enseñanza de la Geografía, Objetivos de Gestión de la UPNFM, Lineamientos Estratégicos del Plan de Nación y Áreas Temáticas del CSUCA, con el propósito de difundirlas académicamente, tanto nivel interno y externo de la universidad. 


\section{Bibliografía}

Anhert, F. 1980. A note on measurements and experiemnts in geomorphology.

García Ballesteros, Aurora. Et.al.1998. Métodos y Técnicas Cualitativas en Geografía Social. Editorial Oikos Tan. España.

Martínez Llantada, Marta. 2005. Metodología de la Investigación Educacional. "Enfoques actuales de la investigación Educativa. Segunda Edición. Editorial Pueblo y Educación. La Habana, Cuba.

Muñoz Campos, Roberto. 2000. La Investigación Científica Paso a Paso. Talleres gráficos UCA. Reimpresión El Salvador

Ortez, Eladio Zacarías. 2000. Así se Investiga. Editorial Clásicos Boxsil, Santa Tecla, El Salvador.

Sanjaume, María Sala. 1998. Teoría y Métodos en Geografía Física. Editorial Síntesis. Madrid, España. 